\title{
Xanthohumol targets the ERK1/2-Fra1 signaling axis to reduce cyclin D1 expression and inhibit non-small cell lung cancer
}

\author{
FENG GAO $^{1,2^{*}}$, MING LI $^{1,3,4^{*}}$, LI ZHOU $^{5 *}$, WENBIN LIU $^{6}$, HUILAN ZUO $^{1,2}$ and WEI LI ${ }^{1,7}$ \\ ${ }^{1}$ Cell Transplantation and Gene Therapy Institute; ${ }^{2}$ Department of Ultrasonography, \\ The Third Xiangya Hospital of Central South University, Changsha, Hunan $410013 ;{ }^{3}$ Changsha Stomatological Hospital, \\ Changsha, Hunan 410004; ${ }^{4}$ School of Stomatology, Hunan University of Chinese Medicine, Changsha, Hunan 410208; \\ ${ }^{5}$ Department of Pathology, Xiangya Hospital of Central South University; ${ }^{6}$ Department of Pathology, Hunan Cancer Hospital; \\ ${ }^{7}$ Department of Radiology, The Third Xiangya Hospital of Central South University, Changsha, Hunan 410013, P.R. China
}

Received February 25, 2020; Accepted June 5, 2020

DOI: 10.3892/or.2020.7697

\begin{abstract}
High expression of cyclin D1 has a crucial role in the maintenance of unlimited cell growth in human cancer cells. The present study indicated that cyclin D1 was overexpressed in human non-small cell lung cancer (NSCLC) tumor tissues and cell lines. Knockout of cyclin D1 suppressed NSCLC cell growth, colony formation and in vivo tumor growth. Of note, the natural product xanthohumol (Xanth) inhibited NSCLC cells via the downregulation of cyclin D1. A further mechanistic study revealed that Xanth suppressed ERK1/2 signaling and reduced the protein levels of FOS-related antigen 1 (Fra1), which eventually inhibited the transcriptional activity of activator protein-1 and decreased the mRNA level of cyclin D1. Furthermore, suppression of ERK1/2 impaired Fra1 phosphorylation and enhanced Xanth-induced Fra1 ubiquitination and degradation. In addition, the S265D mutation compromised Xanth-induced Fra1 degradation. Finally, the in vivo anti-tumor effect of Xanth was validated in a xenograft mouse model. In summary, the present results indicated that targeting ERK1/2-Fra1-cyclin D1 signaling is a promising anti-tumor strategy for NSCLC treatment.
\end{abstract}

\section{Introduction}

Non-small cell lung cancer (NSCLC) is one of the most common types of human lung malignancies and its incidence and mortality have increased over the past decades worldwide $(1,2)$. Although surgical treatment is the mainstay for

Correspondence to: Dr Wei Li, Department of Radiology, The Third Xiangya Hospital of Central South University, 138 Tongzipo Road, Changsha, Hunan 410013, P.R. China

E-mail:weililx@csu.edu.cn

*Contributed equally

Key words: non-small cell lung cancer, xanthohumol, Fra1, cyclin D1 early cases, NSCLC is frequently diagnosed at an advanced stage and metastasis and relapse remain the major causes of therapy failure (3). Accumulating evidence has revealed that activating mutations of oncogenes, including epidermal growth factor receptor (EGFR), K-Ras and c-Met, are a driving force to contribute to the tumorigenesis of certain NSCLCs (4). Targeting therapies towards such driving mutations have emerged as first-line therapeutic strategies for advanced NSCLC. However, most patients who initially respond to targeted therapies eventually develop acquired resistance $(5,6)$. Further elucidation of the underlying mechanisms of resistance and identification of novel anti-tumor agents for NSCLC treatment is necessary.

Cell cycle progression and transitions are tightly controlled by cyclins and cyclin-dependent kinases (CDKs). In mammalian cells, three isoforms of cyclin D (D1, D2 and D3) have been identified $(7,8)$. The complex formed by cyclin $\mathrm{D}$ with either CDK4 or CDK6 is required for retinoblastoma protein phosphorylation and subsequent G1 to S phase progression $(7,9)$. Numerous studies revealed that cyclin D, particularly cyclin D1, is overexpressed in multiple types of human cancer. Given the role of cyclin D1 in mediating extracellular signaling with cell proliferation, it is not surprising that overexpression of cyclin D1 and hyperactivation of their cognate CDK kinases directly contribute to unlimited neoplastic growth $(10,11)$. Thus, the development of novel inhibitors targeting cyclin D1-CDK4/6 signaling is a promising antitumor strategy for clinical treatment.

In the present study, cyclin D1 was determined to be overexpressed in human NSCLC tissues and cell lines. Furthermore, a natural product, Xanthohumol (Xanth), was identified as a potential anti-tumor agent for NSCLC treatment. The inhibitory effect of Xanth on NSCLC cells was determined in vitro and in vivo and the underlying mechanism of the Xanth-mediated anti-tumor activity was investigated.

\section{Materials and methods}

Cell culture and antibodies. The natural product Xanth, proteasome inhibitor MG132 and cycloheximide were purchased from Selleck Chemicals. Cell culture medium and 
supplements, including Dulbecco's modified Eagle's medium (DMEM), RPMI-1640 and fetal bovine serum (FBS), were obtained from Invitrogen (Thermo Fisher Scientific, Inc.). Human NSCLC cell lines, including H520, H358, H1299, H23, HCC 827 and H1975, were purchased from the American Type Culture Collection (ATCC). The H520 is an EGFR null cell, HCC827 (E746-A750 deletion) and H1975 (L858R/T790M) are two NSCLC cells with EGFR activation mutation, while H1299 and H23 are wild EGFR harbor cells. As EGFR signaling plays a crucial role in NSCLC, cells with wild-type EGFR or activation mutant were selected in the present study. The immortalized human lung epithelial cell lines HBE and NL20 were obtained from Sigma and ATCC, respectively. All the cells were maintained in an incubator at $37^{\circ} \mathrm{C}$ in a humidified atmosphere with $5 \% \mathrm{CO}_{2}$ according to the ATCC protocols and subjected to a mycoplasma test every two months. The primary antibodies to cyclin D1, c-Jun, Jun B, Jun D, Fos B, FOS-related antigen 1 (Fra1), phosphorylated (p)-Fra1, c-Fos, p-ERK1/2 and $\beta$-actin were obtained from Cell Signaling Technology, Inc. The anti-Ki67 antibody for immunohistochemistry (IHC) was a product of Abcam. Antibody conjugates were visualized by chemiluminescence (Thermo Fisher Scientific, Inc.). The jetPEI (Qbiogene, Inc.) was used for transient transfection following the standard protocol.

MTS assay. The MTS assay was performed as previously described (12). In brief, NSCLC cells were seeded in 96-well plates $(3,000$ cells/well) and maintained for $24 \mathrm{~h}$. The cells were treated with various doses of Xanth for $72 \mathrm{~h}$. The cell viability was examined using the MTS Cell Proliferation Assay kit (cat. no. G3580; Promega Corp.) following the standard protocol.

Soft agar assay. The soft agar assay for colony formation was performed as described previously (13). In brief, $3 \mathrm{ml}$ Eagle's basal medium containing $0.6 \%$ agar, $10 \%$ FBS and various doses of Xanth was used for the agar base in a 6-well plate. NSCLC cells were counted and suspended at a concentration of 8,000 cells $/ \mathrm{ml}$ using Eagle's basal medium containing $0.3 \%$ agar, 10\% FBS and Xanth. The re-suspended cells were overlaid into a 6-well plate with a $0.6 \%$ agar base and maintained for 2 weeks. The number of colonies was counted under a light microscope (Olympus).

Flow cytometry. Flow cytometric analysis was performed as described previously (14). In brief, the cells were treated with Xanth or DMSO (control) as indicated. Cells were suspended at a concentration of $1 \times 10^{6}$ cells $/ \mathrm{ml}$ with PBS. The propidium iodide staining buffer containing RNase was added to the cell suspension, followed by incubation at room temperature for $15 \mathrm{~min}$ in the dark. The cells were washed with PBS and analyzed with a FACSort flow cytometer (BD Biosciences).

Dual reporter assay. The dual reporter assay was performed as described previously (15). In brief, the Renilla luciferase reporter construct pRL-SV40 was co-transfected with the pGL3-Basic control or the pGL3-AP-1 (cat. no. 40342; Addgene) construct which contain three canonical AP-1 binding sites (TGACTCA) into human NSCLC cells using Lipofectamine 2000 (Thermo Fisher). The transfected cells were treated with Xanth for another $24 \mathrm{~h}$. Cell lysates were prepared using the Dual-Luciferase Reporter Assay kit (cat. no. E1910; Promega Corp.) and subjected to luciferase activity analysis. Renilla luciferase activity was used as an internal control for normalization.

$R T-q P C R$. NSCLC cells were treated with Xanth for $24 \mathrm{~h}$, total RNA was extracted using the Absolutely RNA ${ }^{\circledR}$ Purification Kits (Agilent). SYBR ${ }^{\circledR}$-Green Quantitative RT-qPCR Kit was used in RT-qPCR. Amplification cycles were $95^{\circ} \mathrm{C}$ for $10 \mathrm{~min}$, followed by 40 cycles of $95^{\circ} \mathrm{C}$ for $15 \mathrm{sec}$ and $55^{\circ} \mathrm{C}$ for $60 \mathrm{sec}$ ). The RT-qPCR results were normalized to $\beta$-actin. Cyclin D1 primer sequences used were: Forward, TCTACACCGACA ACTCCATCCG; reverse, TCTGGCATTTTGGAGAGG AAGTG.

Clinical tissue sample collection. All the surgical specimens were collected in accordance with an Institutional Review Board-approved protocol. NSCLC tissues and matched adjacent non-tumor tissues were collected from 35 patients who provided written informed consent at the Department of Pathology, Hunan Cancer Hospital of Central South University (Changsha, China). The in vivo experiments were approved by the Institutional Animal Care and Use Committee of Central South University (Changsha, China).

Western blot analysis. The whole-cell extract (WCE) was prepared using the commercial radioimmunoprecipitation assay buffer (cat. no. PI89901; Thermo Fisher Scientific, Inc.) and the protein concentration was determined by the bicinchoninic acid protein assay (cat. no. 23228; Thermo Fisher Scientific, Inc.). For EGF stimulation, NSCLC cells were starved with $0.1 \%$ fetal bovine serum in RPMI-1640 medium overnight and pretreated with Xanth for $2 \mathrm{~h}$. EGF $(50 \mathrm{ng} / \mathrm{ml})$ was added to the cell culture medium and maintained for $1 \mathrm{~h}$, whole cell lysates were collected for immunoblotting (IB) as described previously (16). In brief, WCE $(20 \mu \mathrm{g})$ was boiled with loading buffer for $5 \mathrm{~min}$ at $95^{\circ} \mathrm{C}$ and subjected to SDS-PAGE, followed by electrotransfer to the polyvinylidene difluoride membrane. The membrane was blocked with $5 \%$ non-fat milk, followed by incubation with primary and secondary antibodies, respectively. The antibodies against cyclin D1 (cat. no. 55506, 1:1,000), p21 (cat. no. 2947, 1:1000), p27 (cat. no. 3686, 1:1,000), c-Jun (cat. no. 9165, 1:1,000), JunB (cat. no. 3753, 1:1,000), JunD (cat. no. 5000, 1:1,000), FosB (cat. no. 2251, 1:1,000), Fra1 (cat. no. 5281, 1:1,000), c-Fos (cat. no. $2250,1: 1,000$ ), p-ERK1/2 (cat. no. 4370, 1:1,000), pP90RSK (cat. no. 8753,1:1,000), p-Fral (cat. no. 5841, 1:1,000), Ubiquitin (cat. no. 3936, 1:1,000), Ki67 (cat. no. 9027, 1:1,000), anti-mouse IgG HRP-linked antibody (cat. no. 7076, 1:10,000), and anti-rabbit IgG, HRP-linked antibody (cat. no. 70764, 1:10,000), were purchased from Cell Signaling Technology (Danvers, MA). The target protein was visualized by chemiluminescence.

Generation of stable cyclin D1 knock-out cell lines. CRISPR-Cas9-mediated gene knockout was performed as previously described (17). In brief, single-guide (sg)RNAs (\#1, GTTCGTGGCCTCTAAGATGA; \#2, GAAGCGTGT GAGGCGGTAGT) targeting cyclin D1 were used for the construction of stable cell lines. In brief, NSCLC cells were transfected with cyclin D1 sgRNA and selected by $1 \mu \mathrm{g} / \mathrm{ml}$ 
puromycin for three weeks. Single colonies were chosen for further study.

In vivo tumor growth. The in vivo experiments were approved by the Institutional Animal Care and Use Committee of Central South University (Changsha, China). Mice were kept in colony cages with free access to food and tap water and in standardized housing conditions (natural $12 \mathrm{~h}$ light-dark cycle, temperature of $23 \pm 1^{\circ} \mathrm{C}$, relative humidity of $55 \pm 5 \%$ ). The proper care and use of experimental animals, including efforts to minimize suffering and distress, use of analgesics or anaesthetics, was based on the Guide for the Care and Use of Laboratory Animals (National Academies Press, Washington, DC). The xenograft model was constructed by subcutaneous injection of HCC 827 cells $\left(4 \times 10^{6}\right)$ into the right flank of 6 -week-old athymic nude mice $(n=5)$. Compound treatment was initiated when the tumor reached an average volume of $100 \mathrm{~mm}^{3}$. The compound-treated group was administered Xanth $(10 \mathrm{mg} / \mathrm{kg})$ every two days by i.p. injection. The health and behaviour of mice were monitored every two days. The tumor volume was determined according to the following formula: Lengthxwidth $2 / 2$. The tumor-bearing mouse was euthanized by $\mathrm{CO}_{2}$ when the tumor volume reached $700 \mathrm{~mm}^{3}$ (32 days). The fill rate of carbon dioxide is $30 \%$ of the chamber volume per minute (3 liter/min), and the duration time is $5 \mathrm{~min}$. Death was further confirmed by cervical dislocation.

IHC. Tumor tissues from clinical samples and mouse xenograft tumors were fixed and subjected to IHC analysis as previously described (18). In brief, the tissue slides were deparaffinized and rehydrated by consecutive incubation with xylene and ethanol, followed by submerging in sodium citrate buffer (10 mM, pH 6.0) and boiling for $10 \mathrm{~min}$ for antigen retrieval. The activity of endogenous peroxidase was quenched by incubation with $3 \% \mathrm{H}_{2} \mathrm{O}_{2}$ in methanol for $10 \mathrm{~min}$. Tissue slides were washed with PBS and blocked with $50 \%$ goat serum albumin. After incubation with the primary and secondary antibodies, the target protein was visualized using 3,3'-diaminobenzidine substrate and samples were counterstained with hematoxylin.

Statistical analysis. Statistical analysis was performed using GraphPad Prism 5.0 (GraphPad, Inc.). Quantitative data are expressed as the mean \pm standard deviation. The significance of differences between groups was evaluated using the Student's t-test or One-way ANOVA. Dunnett's method was used to compare treatment groups to a control group, and Tukey's method was used to compare each group with every other group. $\mathrm{P}<0.05$ was considered to indicate a statistically significant difference.

\section{Results}

High expression of cyclin D1 is required for the maintenance of tumorigenic properties of NSCLC cells. To determine the oncogenic function of cyclin D1 in NSCLC, the protein levels of cyclin D1 were first examined in tumor tissues. As shown in Fig. 1A, cyclin D1 was significantly upregulated in human NSCLC tissues compared with that in the paired non-tumor adjacent tissues. The IB results suggested that cyclin D1 was overexpressed in all human NSCLC cancer cell lines assessed: H520, H358, H1299, H23, HCC827 and H1975 (Fig. 1B). Furthermore, stable cyclin D1-knockout cell lines were constructed from HCC827 and H1975 cells. The MTS data revealed that the viability of cells with stable expression of sgCyclin D1 was significantly reduced (Fig. 1C). To determine the role of cyclin D1 in anchorage-independent growth of NSCLC cells, colony formation was examined using the soft agar assay. The results suggested that knockout of cyclin D1 inhibited colony formation of $\mathrm{H} 1975$ and HCC827 cells, as the colony number was decreased $>60 \%$ when compared to that of cyclin D1-proficient cells (Fig. 1D). The results of the in vivo tumor growth experiment indicated that depletion of cyclin D1 inhibited in vivo tumor development in the xenograft mouse model. The sgCyclin D1-expressing HCC827 xenograft tumors exhibited a reduced tumor volume (Fig. 1E) and a smaller tumor size (Fig. 1F and G). These results suggested that cyclin D1 is highly expressed in NSCLC tissues and cell lines, while knockout of cyclin D1 decreased the tumorigenic properties of NSCLC cells.

Xanth inhibits NSCLC cells in vitro. Accumulating evidence indicates that the natural product Xanth (Fig. 2A) has potent anti-tumor activity against multiple human cancer cell types (19). However, the anti-tumor effect of Xanth on NSCLC cells and the underlying mechanisms have remained largely elusive. The present results indicated that Xanth exhibited only slight cytotoxic effects on the immortalized lung epithelial cell lines HBE and NL20, as the cell viability was not significantly reduced after treatment with Xanth (Fig. 2B). However, the viability of Xanth-treated NSCLC cell lines, including HCC827, H1975 and H23, was decreased by Xanth in a dose-dependent manner (Fig. 2C). Treatment with Xanth for $72 \mathrm{~h}$ reduced the cell viability $>60 \%$ in all treated NSCLC cell lines. Furthermore, the results of the soft agar colony formation assay suggested that the anchorage-independent cell growth of NSCLC cells was significantly reduced with Xanth treatment (Fig. 2D). The Xanth exerted its inhibitory effect on colony formation in a dose-dependent manner. The results revealed that treatment with $10 \mu \mathrm{M}$ Xanth decreased the colony number $>95 \%$ in HCC $827, \mathrm{H} 1975$ and $\mathrm{H} 23$ cells (Fig. 2D). These in vitro results indicated that Xanth inhibits NSCLC cells, but not the immortalized lung epithelial cells, in a dose-dependent manner.

Xanth reduces the protein levels of cyclin DI and suppresses Fral in NSCLC cells. As Xanth exhibited a significant inhibitory effect on NSCLC cell growth, it was then determined whether Xanth affected cell cycle-associated proteins. The IB data revealed that Xanth reduced the protein levels of cyclin D1 in HCC827, H1975 and H23 cells (Fig. 3A). Furthermore, flow cytometric analysis revealed that Xanth induced cell cycle arrest at the G0/G1 phase in HCC827 cells (Fig. 3B). Consistently with this, the IB results revealed that Xanth increased the protein levels of p21 and p27 (Fig. 3C). To investigate the underlying mechanisms of how Xanth reduced cyclin D1 at the protein level, Xanth-treated NSCLC cells were subjected to RT-qPCR analysis of cyclin D1 expression. As shown in Fig. 3D, Xanth decreased the mRNA levels of cyclin D1 in a dose-dependent manner, indicating that Xanth suppressed cyclin D1 transcription. The AP-1 protein is one 
A
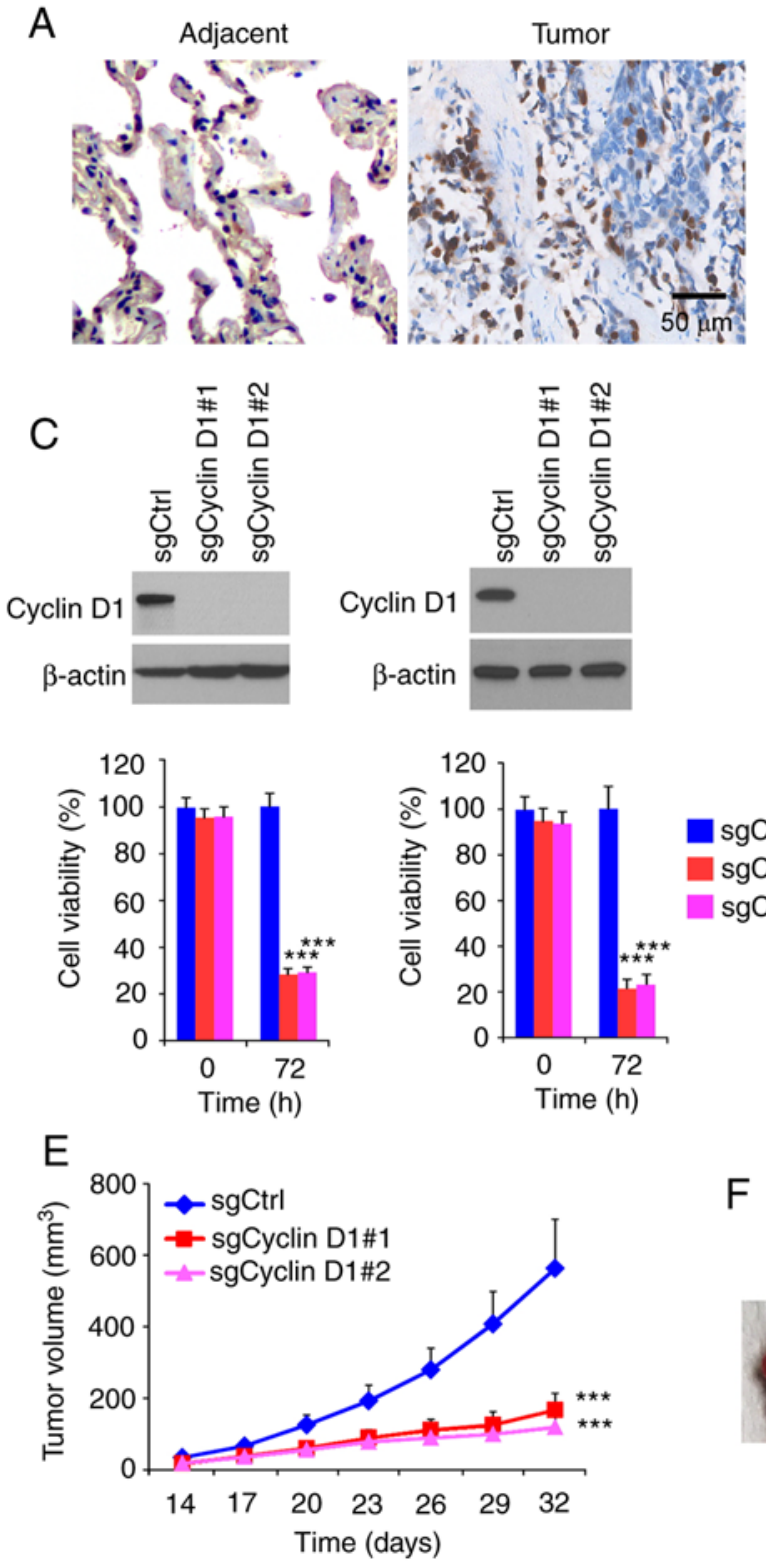

Cyclin D1 expression

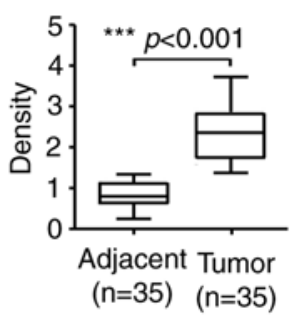

B

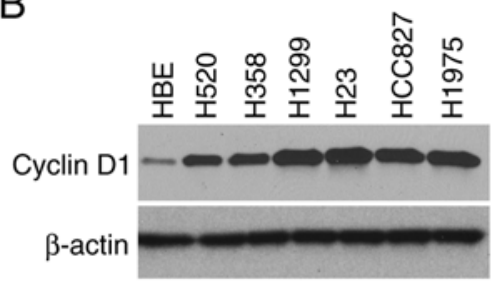

D
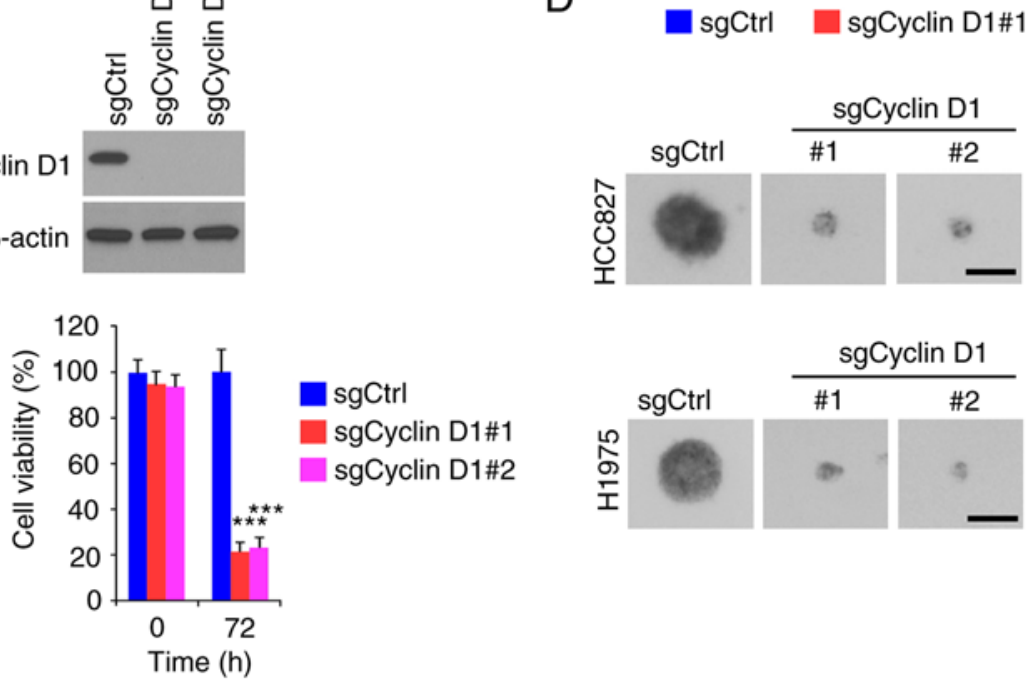

sgCyclin D1\#2
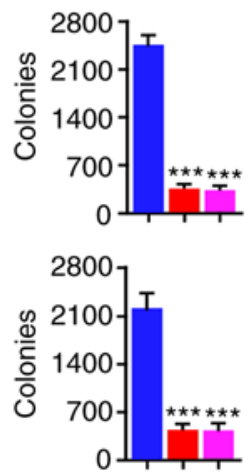

$\mathrm{F}$

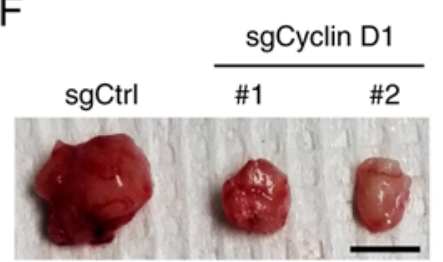

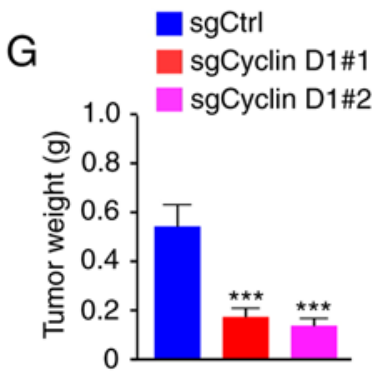

Figure 1. Cyclin D1 is highly expressed in NSCLC cells and required for the malignant phenotype. (A) IHC staining analysis of cyclin D1 expression in 30 cases of matched NSCLC patient tissues and adjacent tissues. Left panel, representative IHC staining results of cyclin D1 (scale bar, $50 \mu \mathrm{m}$ ). Right panel, IHC score. (B) IB analysis of cyclin D1 expression in immortalized lung epithelial cells and NSCLC cells. (C) Cell viability of HCC827 (left) and H1975 (right) cells expressing sgCtrl or sgCyclin D1. Top, IB analysis of cyclin D1 expression. Bottom, cell viability assessed by an MTS assay. (D) Colony formation of HCC827 and H1975 cells expressing sgCtrl or sgCyclin D1 (scale bar, $200 \mu \mathrm{m}$ ). (E-G) In vivo tumor growth of HCC827 cells expressing sgCtrl or sgCyclin D1: (E) Tumor volume, (F) images of the tumors (scale bar, $1 \mathrm{~cm}$ ), (G) tumor weight. ${ }^{* * * *} \mathrm{P}<0.001$. NSCLC, non-small cell lung cancer; IHC, immunohistochemistry; IB, immunoblot; Ctrl, control; sgCyclin D1, single-guide RNA targeting cyclin D1.

of the most important transcription factors required for cyclin D1 transcription (20). The present results suggested that Xanth slightly decreased the protein levels of JunD but robustly reduced the expression of Fra1 in HCC 827 and H1975 (Fig. 3E) cells. Furthermore, the reporter assay indicated that Xanth significantly reduced the luciferase activity of AP-1 (Fig. 3F and G). These results suggested that Fra1 is a critical transcription factor for cyclin D1 expression in NSCLC cells. Indeed, ectopic overexpression of Fra1 in HCC827 (Fig. 3H) and H1975 (Fig. 3I) cells compromised Xanth-induced cyclin D1 downregulation. Consistently, the RT-qPCR results revealed that overexpression of Fra1 restored cyclin D1 mRNA levels in Xanth-treated NSCLC cells (Fig. 3J). Overall, these results suggest that Xanth reduces the protein levels of cyclin D1 in a Fra1-dependent manner in NSCLC cells.

Inhibition of ERK1/2 signaling is required for Xanth-induced Fral reduction in NSCLC cells. To determine the underlying mechanisms of how Xanth decreases the protein levels of Fra1, the signaling transduction in Xanth-treated NSCLC cells was examined. Of note, the IB data suggested that Xanth inhibited the activation of ERK1/2 signaling in a dose-dependent manner. The phosphorylation of ERK1/2 and the downstream target kinase P90RSK was robustly decreased after Xanth treatment (Fig. 4A). Furthermore, Xanth attenuated EGF-induced ERK1/2 and P90RSK phosphorylation in 
A<smiles>COc1cc(/C=C/C(=O)N2CCC=CC2=O)cc(OC)c1OC</smiles>

C

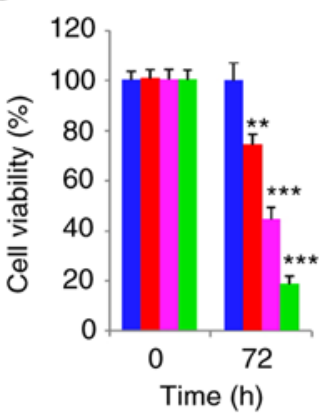

D

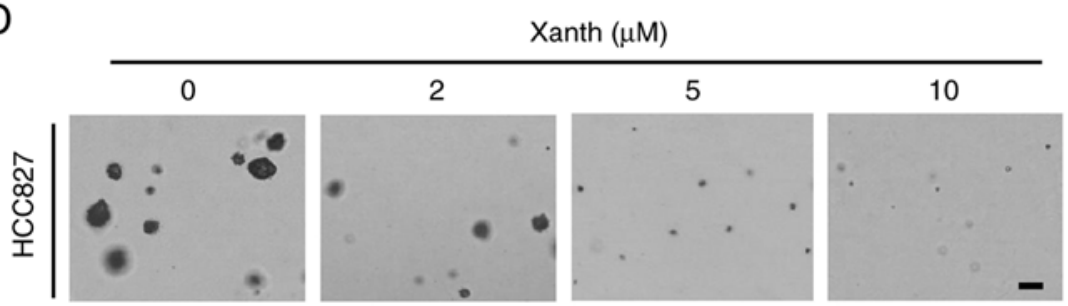

Xanth $(\mu \mathrm{M})$

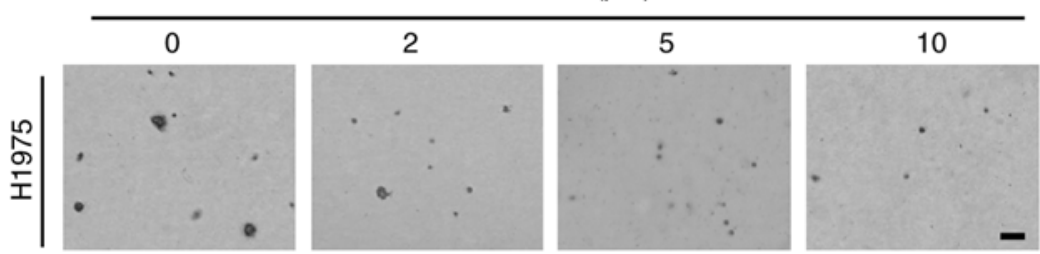

Xanth $(\mu \mathrm{M})$

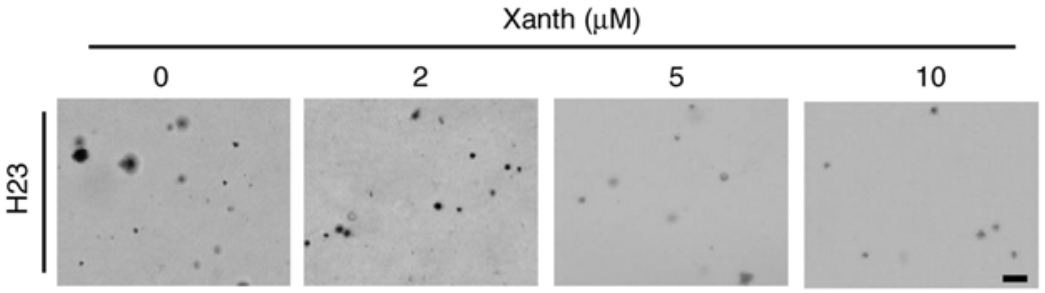

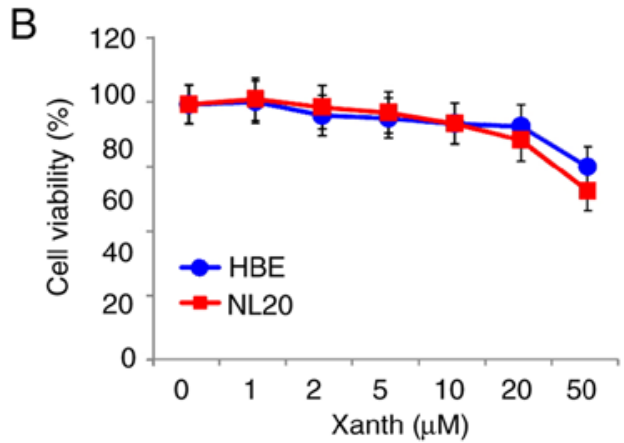
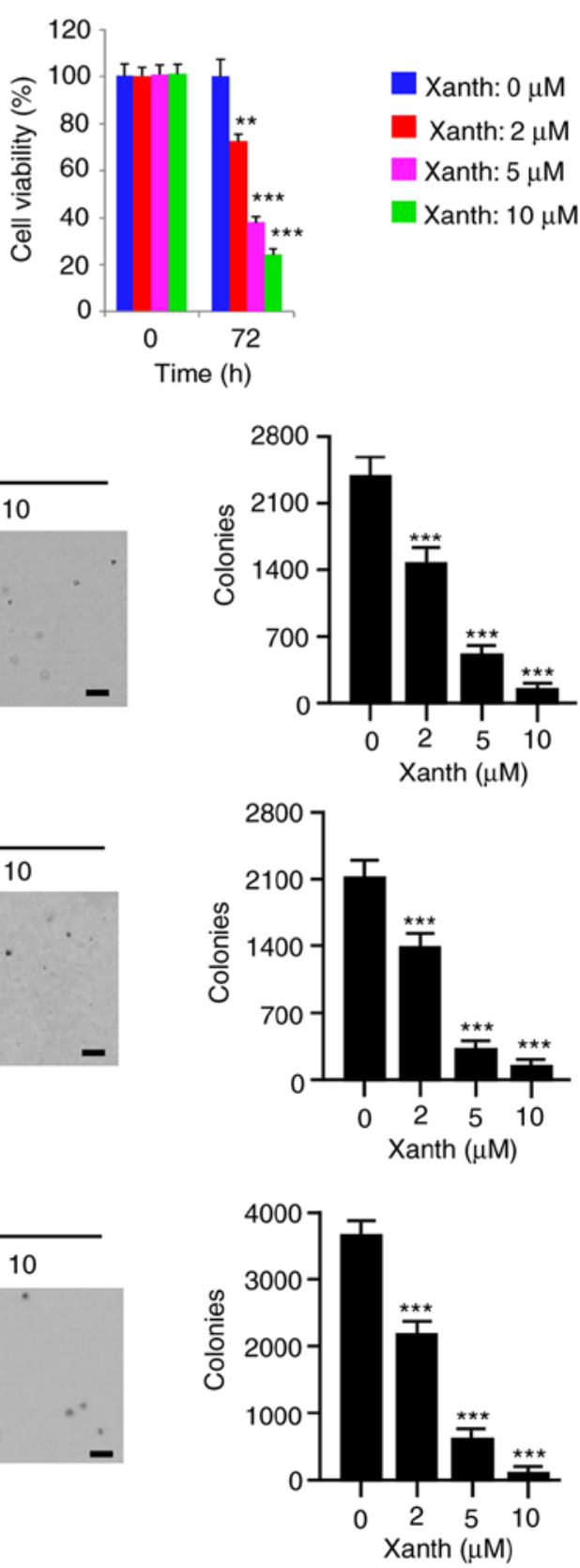

Figure 2. Xanth inhibits the growth of non-small cell lung cancer cells in vitro. (A) Chemical structure of Xanth. (B) Analysis of the toxicity of Xanth on immortalized lung epithelial cell lines HBE and NL20. HBE and NL20 cells were treated with Xanth for $72 \mathrm{~h}$. Cell viability was analyzed by the MTS assay. (C) MTS analysis of the inhibitory effect of Xanth on HCC827 (left), H1975 (middle) and H23 (right) cells. (D) A soft agar assay was performed to assess the inhibitory effect of Xanth on HCC827 (top), H1975 (middle) and H23 (bottom) cells (scale bar, 200 $\mu \mathrm{m}$ ). ${ }^{*} \mathrm{P}<0.05$, ${ }^{* *} \mathrm{P}<0.01,{ }^{* * * *} \mathrm{P}<0.001 . \mathrm{X}^{2} \mathrm{anth}, \mathrm{xanthohumol}$.

NSCLC cells (Fig. 4B). Treatment with the ERK1/2 signaling inhibitor PD98059 suppressed the protein levels of Fra1 and cyclin D1 in HCC827, H1975 and H23 cells. In addition, the phosphorylation of Fra1 on S265, a phosphorylation residue that is catalyzed by ERK1/2, was inhibited in response to PD98059 treatment (Fig. 4C). A previous study suggested that 
A

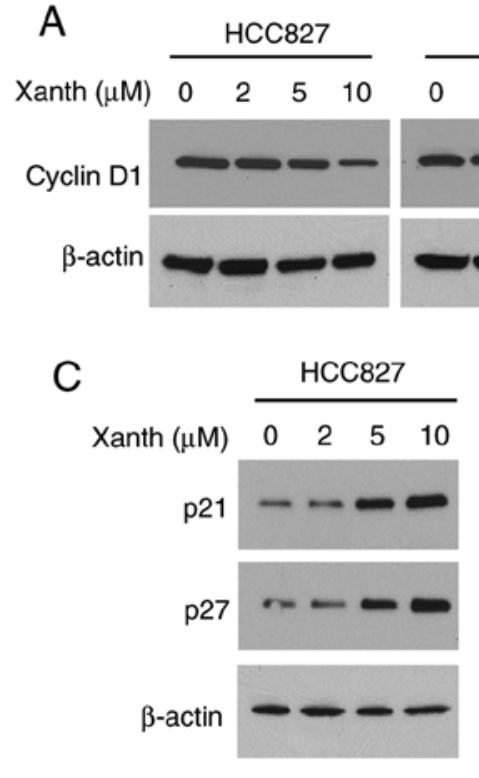

E

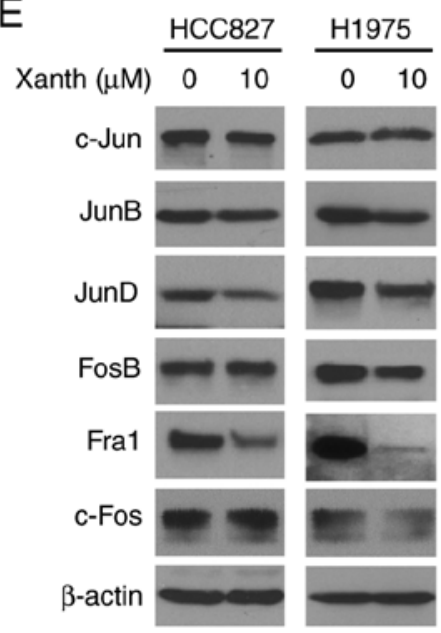

$\mathrm{H}$

Fra1 - -+

Xanth $(\mu \mathrm{M}) \quad 0 \quad 10 \quad 10$

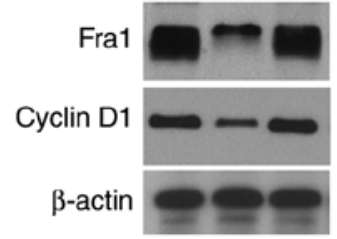

1975
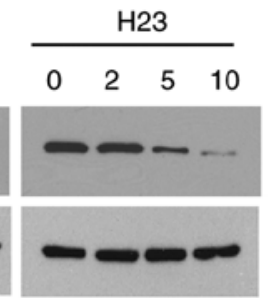

B

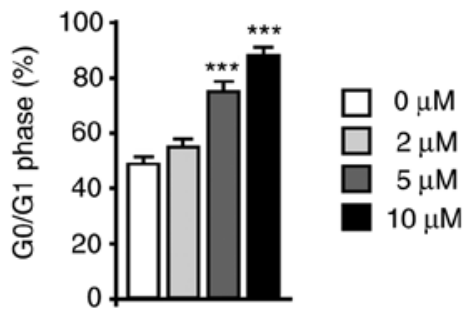

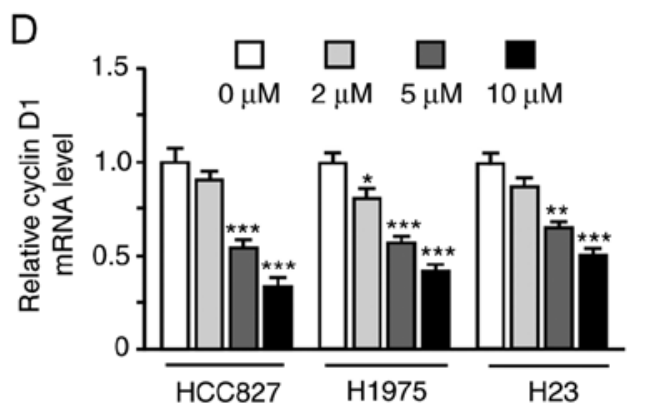
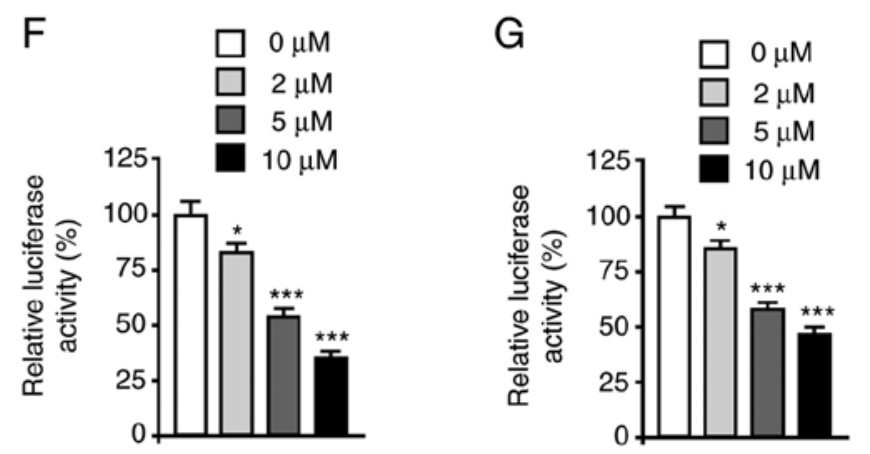

$J$

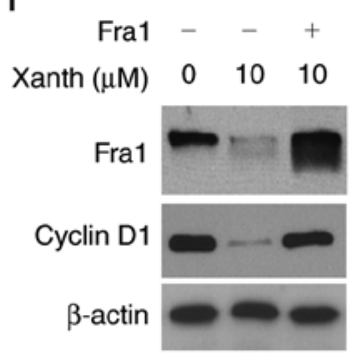

Figure 3. Xanth reduces the protein levels of cyclin D1 and suppresses Fra1 in NSCLC cells. (A) NSCLC cells were treated with Xanth for $24 \mathrm{~h}$ and subjected to IB analysis. (B) HC827 cells were treated with PL for $24 \mathrm{~h}$ and subjected to cell cycle analysis by flow cytometry. (C) HCC 827 cells were treated with Xanth for $24 \mathrm{~h}$ and subjected to IB analysis. (D) NSCLC cells were treated with Xanth for $24 \mathrm{~h}$ and subjected to cyclin D1 mRNA analysis by RT-qPCR. (E) HCC827 and H1975 cells were treated with Xanth for $24 \mathrm{~h}$ and subjected to IB analysis. (F) HCC827 and (G) H1975 cells were co-transfected with pGL3-AP-1 or pGL3-Basic control reporter plasmid together with the Renilla luciferase reporter construct pRL-SV40 for $24 \mathrm{~h}$. Cells were then treated with Xanth for another $24 \mathrm{~h}$ and subjected to analysis of luciferase activity. (H) HCC827 and (I) H1975 cells were transfected with Fra1 construct and treated with/without Xanth for $24 \mathrm{~h}$; the cell lysate was then subjected to IB analysis. (J) HCC827 and H1975 cells were transfected with Fra1 construct and treated with/without Xanth for $24 \mathrm{~h}$; cyclin D1 mRNA levels were then examined by RT-qPCR. ${ }^{*} \mathrm{P}<0.05,{ }^{* * *} \mathrm{P}<0.01$ and ${ }^{* * *} \mathrm{P}<0.001$. NSCLC, non-small cell lung cancer; Xanth, xanthohumol; Fra1, FOS-related antigen 1; RT-qPCR, reverse transcription-quantitative PCR; IB, immunoblot.

Fra1 S265 phosphorylation promotes Fra1 stability and inhibits protein degradation (21). Indeed, the IB results suggested that Xanth reduced the half-life of Fral from nearly $2 \mathrm{~h}$ to $40 \mathrm{~min}$ (Fig. 4D). Of note, the proteasome inhibitor MG132 restored
Fra1 protein levels in Xanth-treated NSCLC cells (Fig. 4E), indicating that Xanth promoted the ubiquitination-dependent protein degradation of Fra1 in NSCLC cells. The ubiquitination analysis revealed that Xanth promoted Fra1 ubiquitination 
A

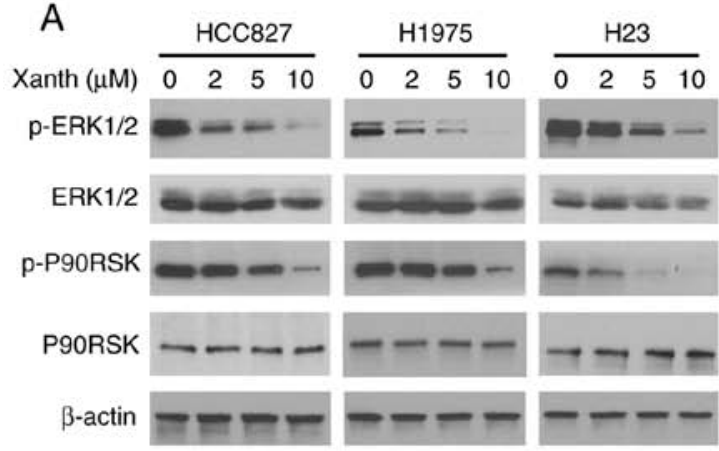

C

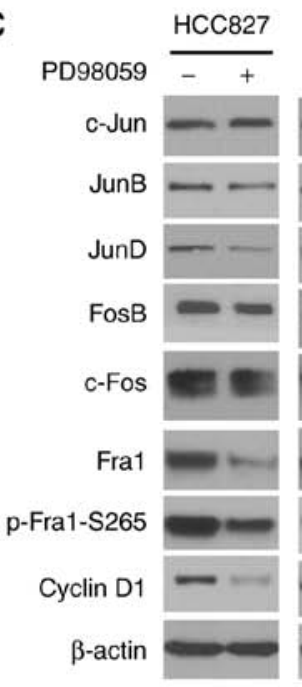

E
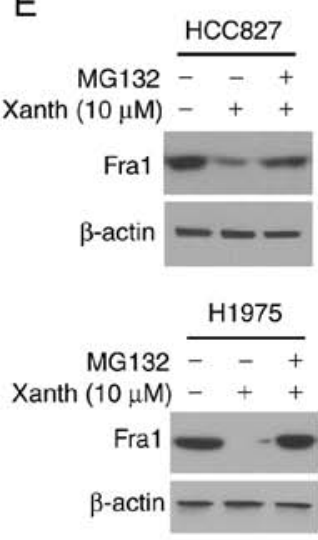

IB: Ub
B

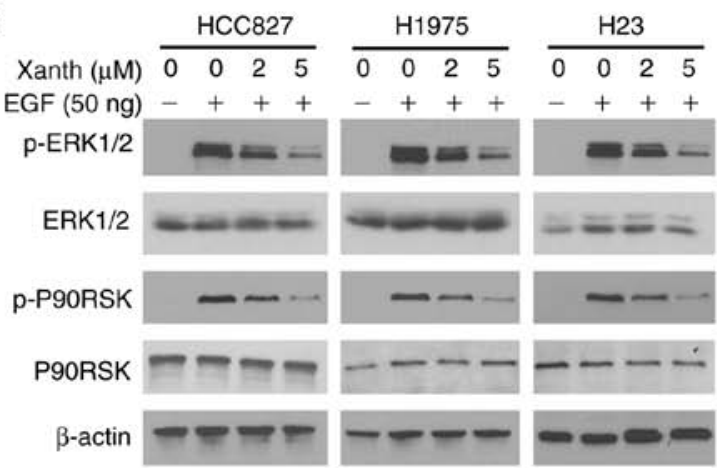

D

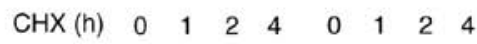

Xanth $(10 \mu \mathrm{M})-\ldots+++$

Fra

$\beta$-actin
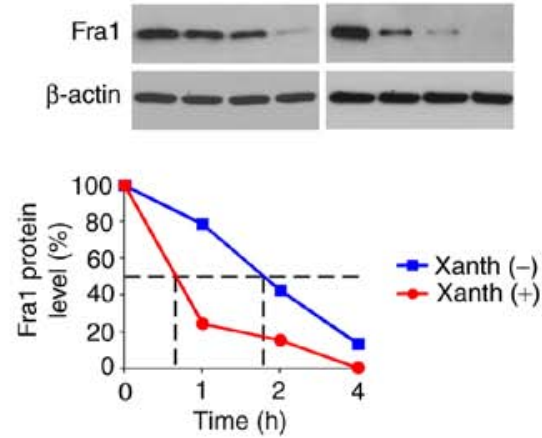

G

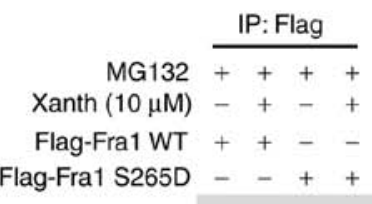

MG132 $\frac{\text { IP: Fra1 }}{++}$

Xanth $(10 \mu \mathrm{M})$ - +

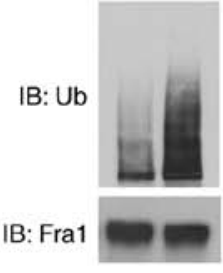

Flag-Fra1 S265D - -++

IB: Ub

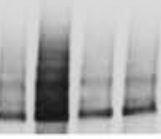

IB: Flag $\rightarrow-\infty=$

Input

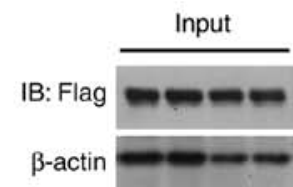

Figure 4. Inhibition of ERK1/2 signaling is required for Xanth-induced reduction of Fra1 in NSCLC cells. (A) NSCLC cells were treated with Xanth for $24 \mathrm{~h}$ and subjected to IB analysis. (B) NSCLC cells were starved with $0.1 \%$ fetal bovine serum in RPMI-1640 medium overnight and pretreated with Xanth for $2 \mathrm{~h}$. EGF $(50 \mathrm{ng} / \mathrm{ml}$ ) was added to the cell culture medium and maintained for $1 \mathrm{~h}$. The cell lysate was subjected to IB analysis. (C) HCC827 (left), H1975 (middle) and H23 (right) cells were treated with PD98059 or DMSO control for $24 \mathrm{~h}$. The whole-cell lysate was collected and subjected to IB analysis. (D) HCC827 cells were treated with/without Xanth for $24 \mathrm{~h}$, and cycloheximide was added to the cell culture medium and maintained for various durations. The cell lysate was subjected to IB analysis. (E) HCC 827 and H1975 cells were treated with Xanth for $24 \mathrm{~h}$, and the proteasome inhibitor MG132 was added to the cell culture medium and cells were maintained for another $6 \mathrm{~h}$. The cell lysate was subjected to IB analysis. (F) HCC827 cells were treated with Xanth for $24 \mathrm{~h}$, the proteasome inhibitor MG132 was added to the cell culture medium and cells were maintained for another $6 \mathrm{~h}$. The cell lysate was subjected to an IP assay with the Fra1 antibody, followed by IB analysis for examination of ubiquitination. (G) HCC827 cells were transfected with various constructs as indicated and treated with Xanth for $24 \mathrm{~h}$. The proteasome inhibitor MG132 was added to the cell culture medium and cells were maintained for another $6 \mathrm{~h}$. The cell lysate was subjected to IP assay with Flag antibody, followed by IB analysis for examination of ubiquitination. Fra1, FOS-related antigen 1; Xanth, xanthohumol; IB, immunoblot; IP, immunoprecipitation.

in HCC827 cells (Fig. 4F). The S265D mutant was then constructed, in which Ser265 was mutated to Asp to mimic the constitutive phosphorylation of Fra1. The results indicated that Xanth promoted the ubiquitination of wild-type Fra1 but not that of the Fra1 S265D mutant (Fig. 4G). Overall, these results suggested that inhibition of ERK1/2-mediated Fra1 S265 phosphorylation is required for Xanth-induced Fra1 destruction in NSCLC cells. 


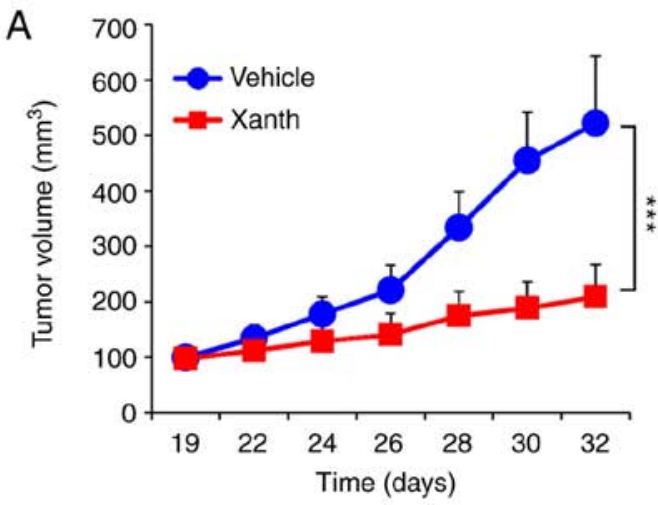

C

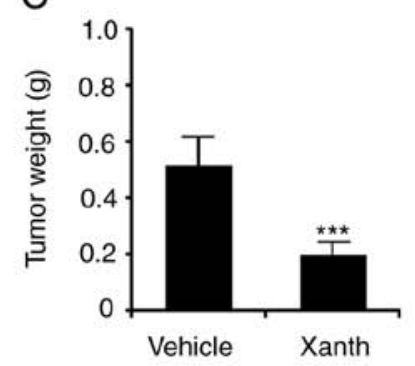

B

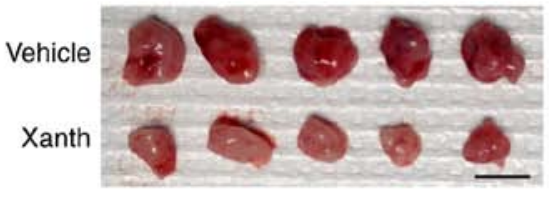

D

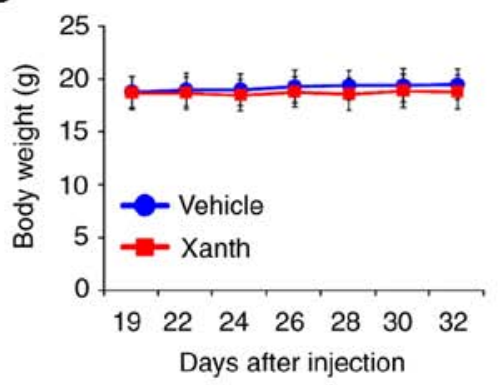

E

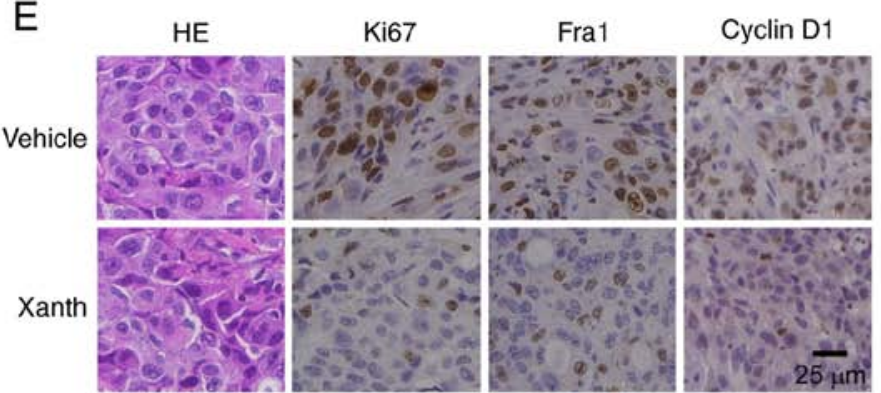

$F$

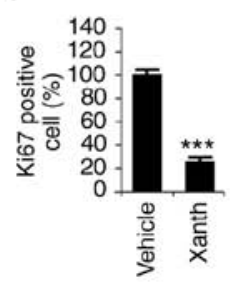

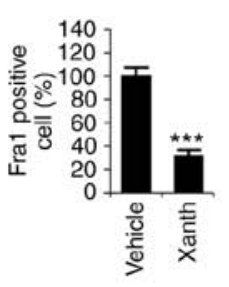

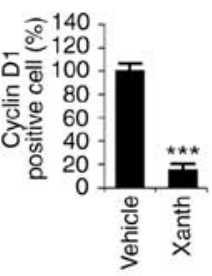

Figure 5. Xanth inhibits the tumor growth of non-small cell lung cancer in vivo. (A) Tumor volume, (B) image of tumor masses and (C) tumor weight of HCC827-derived xenograft tumors in mice treated with vehicle control or Xanth. Scale bar, $1 \mathrm{~cm}$. (D) Body weight of tumor-bearing mice treated with vehicle control or Xanth. (E and F) IHC staining analysis of the expression of Ki67, Fra1 and cyclin D1 in HCC827-derived xenograft tumors with vehicle or Xanth treatment. (E) Representative images of IHC staining (scale bar, $25 \mu \mathrm{m}$ ). (F) IHC score in the different groups. ${ }^{* * *} \mathrm{P}<0.001$. Fra1, FOS-related antigen 1; IHC, immunohistochemistry; Xanth, xanthohumol.

Xanth inhibits the in vivo tumor development of NSCLC cells. To determine the in vivo anti-tumor effect of Xanth, it was tested in a xenograft mouse model established using HCC 827 cells. The results revealed that the average tumor volume of the vehicle-treated group of HCC827-derived tumors was $522 \pm 121 \mathrm{~mm}^{3}$. By contrast, treatment with Xanth significantly suppressed xenograft tumor growth, as the tumor volume was only $209 \pm 58 \mathrm{~mm}^{3}$ (Fig. 5A). Furthermore, Xanth reduced the weight of the tumors $>50 \%$ when compared with that of the vehicle-treated group (Fig. 5B and C). Xanth did not cause a significant body weight loss in the animals (Fig. 5D), suggesting that Xanth was well-tolerated in them. The IHC staining result indicates that Xanth reduced the population of Ki67-positive cells. Similarly, the protein levels of Fra1 and cyclin D1 were significantly downregulated in Xanth-treated xenograft tumors (Fig. 5E and F), which was consistent with the in vitro results suggesting that Xanth reduced the protein levels of Fra1 and cyclin D1. In conclusion, it was indicated that Xanth inhibited the in vivo tumor growth of NSCLC cells in a xenograft mouse model.

\section{Discussion}

The natural product Xanth was previously reported to exhibit a potential anti-cancer effect against multiple human tumor models (19). In vitro and in vivo evidence indicated that Xanth inhibits the growth of colorectal cancer (22), lung cancer (23), prostate cancer (24) and gastric cancer (25). At present, a panel of protein kinases has been identified as potential protein targets of Xanth, including PI3K/Akt (26), mTOR (27) and hexokinase 2 (22). Xanth reduced the malignant phenotypes of human cancer cells to suppress processes including glycolysis (22), angiogenesis and metastasis (28), and to promote apoptosis (29). Although treatment with Xanth induced cell cycle arrest, the underlying mechanism remains elusive. In the present study, Xanth was indicated to inhibit NSCLC cell growth in vitro and in vivo. A further mechanistic study suggested that Xanth downregulated the protein levels of cyclin D1 and induced cell cycle arrest in G0/G1 phase. Of note, it was demonstrated that Xanth reduces the transcription of cyclin D1 in a Fra1-dependent manner. The present data expanded the known anti-tumor mechanisms of Xanth and 
indicate that targeting cyclin D1 and cell cycle progression offers an alternative strategy for NSCLC prevention and treatment.

Cyclin D1 levels may be regulated transcriptionally, translationally and post-translationally (8). Multiple transcription factors have been identified to be required for cyclin D1 transcription, including AP-1, EGFR, NF- $\mathrm{B}$, early growth response 1 and STAT5. A panel of binding consensus for these transcription factors has been identified within the human cyclin D1 promoter region (20). During the past decades, mitogenic growth factors were the most intensively studied stimulators for cyclin D1 transcription and are thought to be among the most important ones. The mitogen-activated protein kinases (MAPKs), such as the Ras-Raf-MAPK kinase-ERK signaling, have a crucial role in mitogenic growth factor-induced cyclin D1 expression (30). Activation of ERK1/2 signaling promotes the expression of AP-1 transcription factors and eventually enhances cyclin D1 transcription $(30,31)$. Cyclin D1 is a nuclear protein. In the present study, IHC data in patient-derived tissues showed that cyclin D1 was highly expressed in tumor tissues. However, in adjacent tissues, there was no significant nuclear staining of cyclin D1. The present results suggested that treatment with Xanth inhibited the activation of ERK1/2 signaling. Consistently, the protein levels of Fral were robustly reduced. The dual reporter assay indicated that Xanth inhibited the transcriptional activity of AP-1, as well as the mRNA expression of cyclin D1. Furthermore, overexpression of Fral compromised this inhibitory effect of Xanth on NSCLC cells. This evidence indicates that targeting the ERK1/2 signaling-mediated AP-1 transcriptional activation contributed to the anti-tumor effect of Xanth.

As an immediate early gene, Fra-1 is frequently overexpressed in human cancers (32). Fra-1 is not able to transform the cells on its own. However, a high expression of Fra1 promotes cell proliferation and survival, as well as angiogenesis of human cancers (32-34). Furthermore, overexpression of Fra1 confers chemo/radioresistance in multiple human cancer models $(35,36)$. The transcription and expression of Fra1 were reported to be increased after stimulation with mitogenic growth factors, including EGF, hepatocyte growth factor and insulin-like growth factor 1 . Activation of ERK1/2 signaling is considered one of the most critical types of upstream signaling of Fral after treatment with mitogenic growth factors (37). Of note, Fra1 is highly expressed in K-Ras-driven cancer cells (38-40). Similar to numerous key cell regulators, the expression of Fral is tightly controlled by protein stability (41). A previous study indicated that ERK1/2 kinase induced the phosphorylation of Fra1 on S265 and compromised ubiquitination-mediated Fral destruction (21). Thus, the hyperactivation of ERK1/2 signaling not only promotes the transcription of Fra1 but also increases protein stability. The present results suggested that Xanth inhibited the activation of ERK1/2 signaling and attenuated Fra1 phosphorylation at S265. The IB data revealed that treatment with Xanth reduced the half-life of Fra1. Treatment with proteasome inhibitor restored the protein levels of Fra1 in Xanth-treated NSLCL cells. Xanth increased the ubiquitination of wild-type Fral but not that of the S265D mutant. The present results demonstrated a novel anti-tumor effect of Xanth and suggested that the inhibitory effect of
Xanth on NSCLC cells was at least partially dependent on Xanth-mediated Fra1 destruction.

In conclusion, the present study suggested that high protein levels of cyclin D1 are required in NSCLC cells for maintaining their malignant phenotype. The natural product Xanth exerted an inhibitory effect on NSCLC cells by decreasing cyclin D1 in a Fra1-mediated, AP-1 transcription activity-dependent manner. Furthermore, it was demonstrated that Xanth inhibited ERK1/2 signaling and Fra1 phosphorylation, which eventually caused ubiquitination-dependent degradation of Fra1. The present study enhanced the understanding of the anti-tumor mechanisms of Xanth and indicated that Xanth is a promising chemotherapeutic agent for NSCLC management.

\section{Acknowledgements}

The authors would like to thank Shiming Tan at the Third Xingya Hospital, for technical support and providing critical comments.

\section{Funding}

This study was supported by the National Natural Science Foundation of China (grant nos. 81904262, and 81972837) and the Natural Science Foundation of Hunan Province (grant nos. 2018JJ3787, 2018JJ2604, and 2019JJ50682).

\section{Availability of data and materials}

All data and materials supporting the conclusion of this study have been included within the article.

\section{Authors' contributions}

FG, ML, LZ, and WL designed the study. HZ, WL, FG, ML, $\mathrm{LZ}$, and WL performed experiments and/or contributed to data analyses. FG, ML, LZ, and WL wrote the manuscript. All authors provided critical review and revisions and approved the final version of the manuscript.

\section{Ethics approval and consent to participate}

The in vivo experiments were approved by the Institutional Animal Care and Use Committee of Central South University (Changsha, China). Written informed consent was provided by patients. There is no human subject participation.

\section{Patient consent for publication}

This study does not include any individual person's data in any form.

\section{Conflicts of interest}

The authors have declared no conflicts of interest.

\section{References}

1. Herbst RS, Morgensztern D and Boshoff C: The biology and management of non-small cell lung cancer. Nature 553: 446-454, 2018. 
2. Qiang H, Chang Q, Xu J, Qian J, Zhang Y, Lei Y, Han B and Chu T: New advances in antiangiogenic combination therapeutic strategies for advanced non-small cell lung cancer. J Cancer Res Clin Oncol 146: 631-645, 2020.

3. Arbour KC and Riely GJ: Systemic therapy for locally advanced and metastatic non-small cell lung cancer: A review. JAMA 322: 764-774, 2019.

4. Skoulidis F and Heymach JV: Co-occurring genomic alterations in non-small-cell lung cancer biology and therapy. Nat Rev Cancer 19: 495-509, 2019.

5. Santoni-Rugiu E, Melchior LC, Urbanska EM, Jakobsen JN, Stricker K, Grauslund M and Sørensen JB: Intrinsic resistance to EGFR-tyrosine kinase inhibitors in EGFR-mutant non-small cell lung cancer: Differences and similarities with acquired resistance. Cancers (Basel) 11: 923, 2019.

6. Shah R and Lester JF: Tyrosine kinase inhibitors for the treatment of EGFR mutation-positive non-small-cell lung cancer: A clash of the generations. Clin Lung Cancer 21: e216-e228, 2020.

7. Qie S and Diehl JA: Cyclin D degradation by E3 ligases in cancer progression and treatment. Semin Cancer Biol: Jan 30, 2020 (Epub ahead of print).

8. Musgrove EA, Caldon CE, Barraclough J, Stone A and Sutherland RL: Cyclin D as a therapeutic target in cancer. Nat Rev Cancer 11: 558-572, 2011.

9. Chou J, Quigley DA, Robinson TM, Feng FY and Ashworth A: Transcription-associated cyclin-dependent kinases as targets and biomarkers for cancer therapy. Cancer Discov 10: 351-370, 2020.

10. Qie S and Diehl JA: Cyclin D1, cancer progression, and opportunities in cancer treatment. J Mol Med (Berl) 94: 1313-1326, 2016.

11. Ramos-García P, González-Moles MÁ, Ayén Á, González-Ruiz L, Gil-Montoya JA and Ruiz-Ávila I: Predictive value of CCND1/cyclin D1 alterations in the malignant transformation of potentially malignant head and neck disorders: Systematic review and meta-analysis. Head Neck 41: 3395-3407, 2019.

12. Zhou Y, Li M, Yu X, Liu T, Li T, Zhou L, Liu W, Li W and Gao F: Butein suppresses hepatocellular carcinoma growth via modulating Aurora B kinase activity. Int J Biol Sci 14: 1521-1534, 2018.

13. Gao F, Yu X, Li M, Zhou L, Liu W, Li W and Liu H: Deguelin suppresses non-small cell lung cancer by inhibiting EGFR signaling and promoting GSK3 $\beta /$ FBW7-mediated Mcl-1 destabilization. Cell Death Dis 11: 143, 2020.

14. Yu X, Liang Q, Liu W, Zhou L, Li W and Liu H: Deguelin, an Aurora B kinase inhibitor, exhibits potent anti-tumor effect in human esophageal squamous cell carcinoma. EBioMedicine 26: 100-111, 2017.

15. Li W, Yu X, Xia Z, Yu X, Xie L, Ma X, Zhou H, Liu L, Wang J, Yang Y and Liu H: Repression of Noxa by Bmil contributes to deguelin-induced apoptosis in non-small cell lung cancer cells J Cell Mol Med 22: 6213-6227, 2018.

16. Liu H, Li W, Yu X, Gao F, Duan Z, Ma X, Tan S, Yuan Y, Liu L, Wang J, et al: EZH2-mediated Puma gene repression regulates non-small cell lung cancer cell proliferation and cisplatin-induced apoptosis. Oncotarget 7: 56338-56354, 2016.

17. Zhou L, Yu X, Li M, Gong G, Liu W, Li T, Zuo H, Li W, Gao F and Liu H: Cdh1-mediated Skp2 degradation by dioscin reprogrammes aerobic glycolysis and inhibits colorectal cancer cells growth. EBioMedicine 51: 102570, 2020.

18. Yu X, Wang R, Zhang Y, Zhou L, Wang W, Liu H and Li W: Skp2-mediated ubiquitination and mitochondrial localization of Akt drive tumor growth and chemoresistance to cisplatin. Oncogene 38: 7457-7472, 2019.

19. Jiang CH, Sun TL, Xiang DX, Wei SS and Li WQ: Anticancer activity and mechanism of xanthohumol: A prenylated flavonoid from Hops (Humulus lupulus L.). Front Pharmacol 9: 530, 2018.

20. Klein EA and Assoian RK: Transcriptional regulation of the cyclin D1 gene at a glance. J Cell Sci 121: 3853-3857, 2008.

21. Basbous J, Chalbos D, Hipskind R, Jariel-Encontre I and Piechaczyk M: Ubiquitin-independent proteasomal degradation of Fra-1 is antagonized by Erk1/2 pathway-mediated phosphorylation of a unique C-terminal destabilizer. Mol Cell Biol 27: 3936-3950, 2007.

22. Liu W, Li W, Liu H and Yu X: Xanthohumol inhibits colorectal cancer cells via downregulation of Hexokinases II-mediated glycolysis. Int J Biol Sci 15: 2497-2508, 2019.

23. Sławińska-Brych A, Zdzisińska B, Dmoszyńska-Graniczka M, Jeleniewicz W, Kurzepa J, Gagoś M and Stepulak A: Xanthohumo inhibits the extracellular signal regulated kinase (ERK) signalling pathway and suppresses cell growth of lung adenocarcinoma cells. Toxicology 357-358: 65-73, 2016.
24. Kłósek M, Mertas A, Król W, Jaworska D, Szymszal J and Szliszka E: Tumor necrosis factor-related apoptosis-inducing ligand-induced apoptosis in prostate cancer cells after treatment with xanthohumol-A natural compound present in Humulus lupulus L. Int J Mol Sci 17: 837, 2016.

25. Wei S, Sun T, Du J, Zhang B, Xiang D and Li W: Xanthohumol, a prenylated flavonoid from Hops, exerts anticancer effects against gastric cancer in vitro. Oncol Rep 40: 3213-3222, 2018.

26. Silva AF, Faria-Costa G, Sousa-Nunes F, Santos MF, Ferreira-Pinto MJ, Duarte D, Rodrigues I, Tiago Guimarães J, Leite-Moreira A, Moreira-Gonçalves D, et al: Anti-remodeling effects of xanthohumol-fortified beer in pulmonary arterial hypertension mediated by ERK and AKT inhibition. Nutrients 11: 583, 2019.

27. Guo D, Zhang B, Liu S and Jin M: Xanthohumol induces apoptosis via caspase activation, regulation of $\mathrm{Bcl}-2$, and inhibition of $\mathrm{PI} 3 \mathrm{~K} / \mathrm{Akt} / \mathrm{mTOR}-\mathrm{kinase}$ in human gastric cancer cells. Biomed Pharmacother 106: 1300-1306, 2018.

28. Saito K, Matsuo Y, Imafuji H, Okubo T, Maeda Y, Sato T, Shamoto T, Tsuboi K, Morimoto M, Takahashi H, et al: Xanthohumol inhibits angiogenesis by suppressing nuclear factor- $\kappa \mathrm{B}$ activation in pancreatic cancer. Cancer Sci 109: 132-140, 2018.

29. Engelsgjerd S, Kunnimalaiyaan S, Kandil E, Gamblin TC and Kunnimalaiyaan M: Xanthohumol increases death receptor 5 expression and enhances apoptosis with the TNF-related apoptosis-inducing ligand in neuroblastoma cell lines. PLoS One 14: e0213776, 2019

30. Chambard JC, Lefloch R, Pouysségur J and Lenormand P: ERK implication in cell cycle regulation. Biochim Biophys Acta 1773: 1299-1310, 2007.

31. Tchakarska G and Sola B: The double dealing of cyclin D1. Cell Cycle 19: 163-178, 2020

32. Dhillon AS and Tulchinsky E: FRA-1 as a driver of tumour heterogeneity: A nexus between oncogenes and embryonic signalling pathways in cancer. Oncogene 34: 4421-4428, 2015.

33. Yun SI, Hong HK, Yeo SY, Kim SH, Cho YB and Kim KK: Ubiquitin-specific protease 21 promotes colorectal cancer metastasis by acting as a Fra-1 deubiquitinase. Cancers (Basel) 12: 207, 2020.

34. Zhang Z, Zhang Y, Zhang L, Pei Y, Wu Y, Liang H, Zhang W and Zhang B: Incomplete radiofrequency ablation provokes colorectal cancer liver metastases through heat shock response by PKC $\alpha$ /Fra-1 pathway. Cancer Biol Med 16: 542-555, 2019.

35. Tyagi A, Vishnoi K, Kaur H, Srivastava Y, Roy BG, Das BC and Bharti AC: Cervical cancer stem cells manifest radioresistance: Association with upregulated AP-1 activity. Sci Rep 7: 4781, 2017.

36. Lu D, Chen S, Tan X, Li N, Liu C, Li Z, Liu Z, Stupack DG, Reisfeld RA and Xiang R: Fra-1 promotes breast cancer chemosensitivity by driving cancer stem cells from dormancy. Cancer Res 72: 3451-3456, 2012.

37. Jiang X, Xie H, Dou Y, Yuan J, Zeng D and Xiao S: Expression and function of FRA1 protein in tumors. Mol Biol Rep 47: 737-752, 2020.

38. Román M,López I, Guruceaga E, Baraibar I,Ecay M, Collantes M, Nadal E, Vallejo A, Cadenas S, Miguel ME, et al: Inhibitor of differentiation-1 sustains mutant KRAS-driven progression, maintenance, and metastasis of lung adenocarcinoma via regulation of a FOSL1 network. Cancer Res 79: 625-638, 2019.

39. Keshamouni VG: Excavation of FOSL1 in the ruins of KRAS-driven lung cancer. Am J Respir Cell Mol Biol 58: 551-552, 2018.

40. Elangovan IM, Vaz M, Tamatam CR, Potteti HR, Reddy NM and Reddy SP: FOSL1 promotes Kras-induced lung cancer through amphiregulin and cell survival gene regulation. Am J Respir Cell Mol Biol 58: 625-635, 2018.

41. Gomard T,Jariel-Encontre I, Basbous J, Bossis G, Moquet-Torcy G and Piechaczyk M: Fos family protein degradation by the proteasome. Biochem Soc Trans 36: 858-863, 2008

This work is licensed under a Creative Commons Attribution-NonCommercial-NoDerivatives 4.0 International (CC BY-NC-ND 4.0) License. 